\title{
Otolaryngology and Covid-19: New Guidelines and Triage for Dealing with the Second Wave
}

\section{Vikas Kakkar ${ }^{1}$, Akriti Sharma ${ }^{2 *}$, Arun Kumar Sharma ${ }^{3}$, Rohit Bhardwaj $^{4}$ and Ritesh Kumar ${ }^{5}$}

${ }^{1}$ Department of Otorhinolaryngology, SGT Medical College, Hospital and Research Institute, Village Budhera, Gurugram, Haryana, India

${ }^{2}$ Assistant Professor, Department of Otorhinolaryngology, SGT Medical College,

Hospital and Research Institute, Village Budhera, Gurugram, Haryana, India

${ }^{3}$ Department of Otorhinolaryngology, SGT Medical College, Hospital and Research

Institute, Village Budhera, Gurugram, Haryana, India

${ }^{4}$ Assistant Professor, Department of Otorhinolaryngology, Army College of Medical

Sciences and Base Hospital, Delhi, India

${ }^{5}$ Assistant Professor, Department of Pharmacology, Department of

Otorhinolaryngology, SGT Medical College, Hospital and Research Institute, Village

Budhera, Gurugram, Haryana, India

*Corresponding Author: Akriti Sharma, Assistant Professor, Department of Otorhinolaryngology, SGT Medical College, Hospital and Research Institute, Village

Budhera, Gurugram, Haryana, India.
Received: October 19, 2021

Published: November 18, 2021

(C) All rights are reserved by Akriti Sharma., et al.

\section{Abstract}

Purpose: Main aim of the study was to provide fresh guidelines for triage of patients during the second wave of covid-19 pandemic, along with laying emphasis upon the need for taking stricter precautions by the healthcare staff in diagnosis and management of patients during this time. These revised guidelines and recommendations, drafted specifically for ENT procedures, have been shared by us after taking into account recently published literature on the second wave of the COVID-19 pandemic put together with our own experience of managing patients in these unprecedented times.

Methods: The ENT Cochrane, PubMed and Web of Science databases were searched without a time limit using the terms 'Covid-19 and SARS-CoV-2 in conjunction with ENT surgery and 'second wave' in India.

Results: Only absolutely essential and emergent procedures need to be carried out with the use of the highest level of Personal protective equipment comprising of powered air-purifying respirators along with eye protection, fluid resistant gowns and disposable aprons and gloves. As RT-PCR alone cannot be relied upon for diagnosis of covid patients, the diagnosis of covid-19 must be based on a comprehensive evaluation taking clinical and radiological findings into account and every patient must be treated as a covid positive patient unless proved otherwise.

Conclusion: Enhanced level of precautions and revised triage strategies would be of immense help to the ENT surgeon in minimizing the exposure to health care workers and patients during the second wave of the covid-19 pandemic. With an additional burden on the Otolaryngologists due to a sudden rise in cases of Mucormycosis and with increasing mortality amongst doctors, protecting the healthcare staff must be an absolute priority.

Keywords: COVID-19; Second Wave; ENT Surgeon; Triage; Precautions 


\section{Introduction}

The COVID-19 outbreak was announced a pandemic by the World Health Organization in March 2020Amongst all healthcare specialists, otolaryngologists were particularly at a high risk of being exposed to the Sars-cov2 virus [1,2] due to its contagious nature and propensity to spread through aerosols. Hence, the first wave of covid -19 necessitated the need for laying down triage strategies and guidelines for otolaryngologists across the world with the aim to protect the healthcare staff without compromising essential patient care. After a cooling period, that lasted a few months following the first wave of the pandemic, a devastating 'second wave' hit India around April 2021. The second wave of covid-19 has proved to be far more deadly with a much higher number of cases than the first wave not only in India but also in Europe and America [3]. Doctors faced multiple challenges while dealing with this pandemic that included working with limited personal protective equipment (PPE), training, rest, and rotation but worst of all loss of life due to coronavirus infection $[4,5]$. Reports claim that at present India is the third worst affected country in the world with more than 2 million people suffering from covid-19 illness and above 43,000 deaths due to the infection [6]. Though physicians from almost all the fields have succumbed to COVID-19 [7], otolaryngologists being in the frontline are at a very high risk and hence need to be extremely cautious. This article has been written with the objective of providing fresh guidelines for triage of patients during the second wave of covid-19 pandemic, keeping in mind that there is a need for taking stricter precautions by the healthcare staff in diagnosis and management of patients during this time. These revised Guidelines and recommendations have been shared by us after taking into account recently published literature on the second wave of the COVID-19 pandemic put together with our own experience of managing patients in these unprecedented times.

\section{Methods}

The ENT Cochrane, Pubmed and Web of Science databases were searched without a time limit using the terms 'Covid-19, SARSCoV-2 and 'second wave' in conjunction with 'ENT surgery' and 'Otolaryngology'.

\section{Comparing the second wave with the first in India}

It is important to understand the fact that the second wave is far more serious and deadly than the first one in India and there could be several reasons for this. Different strains or variants of SARS-
Cov-2 have been reported in literature. The B.1.1.7 strain which was first detected in UK in November 2020, is estimated to be $40-80 \%$ more transmissible than the wild type SARS-CoV-2 [8,9]. Studies have reported that other trains from South Africa (B.1.351), Brazil (P.1), and India (B.1.617) are also much more contagious [10] than the variants in early 2020. One of the important reasons for a second wave in India could be the highly contagious character of B.1.617 which is a double mutant variant of SARS-CoV-2. Other reasons could be negligent behavior of the population, with people not taking adequate precautions and relaxation of interventions in India [11]. While spread of virus through aerosols and respiratory fluids has been documented several times [12-14], recently published literature claims that transmission of SARS-Cov2 happens across distances more than 6 feet [15].

Studies document that the transmission can happen through an infectious person exhaling the virus indoors for a time period that may range from 15 minutes to a few hours leading to the generation of a viral load that is sufficient to transmit infections to people more than 6 feet away, and also sometimes to individuals who have visit the area soon after the infectious person leaves [15].

In our opinion there is an urgent need of a fresh triage strategy particularly for all otolaryngology procedures during this time in order to protect our healthcare staff who are at an increased risk of acquiring COVID-19 due to a much more deadly and contagious strain than before responsible for the second wave of the pandemic in India.

\section{Triage}

During the first wave of Covid-19 in India most triage strategies were centered around performing emergency surgeries while postponing elective ones. A few however believed in categorizing patients into emergent, semi-emergent and elective cases and operating with precautions [16].

With the second wave of the pandemic, hitting the nation like a storm and resulting in increased mortality amongst both, the general population as well as healthcare staff there is an urgent need to device a triage strategy that was stricter and better suited for the current scenario.

In our opinion elective surgeries during the second wave of the pandemic are a complete no. Stricter triage strategies are the need 
of the hour particularly to safeguard the healthcare staff from getting infected. Triage during this time should focus on operating upon absolutely emergent cases.

Hence our only recommendation for triage of ENT patients needing surgical intervention during this time is to operate upon absolute emergencies. Table 1 mentions these emergency conditions with their management in detail.

\begin{tabular}{|c|c|}
\hline $\begin{array}{l}\text { Indications: Only } \\
\text { Emergency cases } \\
\text { requiring intervention } \\
\text { within } 72 \text { hours }\end{array}$ & Management \\
\hline $\begin{array}{l}\text { Foreign bodies of the } \\
\text { airway and esophagus, }\end{array}$ & $\begin{array}{l}\text { Foreign body removal under gen- } \\
\text { eral anesthesia }\end{array}$ \\
\hline Head and Neck Abscesses & Incision and Drainage \\
\hline Uncontrolled epistasis, & $\begin{array}{c}\text { Nasal packing or cauterization in } \\
\text { OT }\end{array}$ \\
\hline $\begin{array}{l}\text { Acute Mastoiditis or other } \\
\text { serious complications of }\end{array}$ & Mastoidectomies \\
\hline $\begin{array}{l}\text { Chronic Suppurative otitis } \\
\text { Media }\end{array}$ & Facial nerve decompression \\
\hline Traumatic facial palsy & $\begin{array}{l}\text { Trachestomies (Excluding the indi- } \\
\text { cation of prolonged intubation) }\end{array}$ \\
\hline $\begin{array}{c}\text { Trauma to face and neck } \\
\text { Airway obstructions }\end{array}$ & $\begin{array}{l}\text { Surgical Debridement in cases of } \\
\text { mucormycosis }\end{array}$ \\
\hline $\begin{array}{l}\text { Malignancies. } \\
\text { Mucormycosis }\end{array}$ & \\
\hline
\end{tabular}

Table 1: Triage of patients during the second wave of Covid 19

$$
\text { Pandemic in India. }
$$

In our opinion, the main triage strategy for surgery during this time should only be to operate upon absolute emergencies mentioned in the table above and resort to conservative management for other conditions as far as possible.

Along with triage strategies even precautions for otolaryngologists should be much stricter than those taken during the first wave.

\section{General considerations for ENT surgeons}

We recommend that, every patient being dealt with by an otolaryngologist should be treated like a COVID positive patient in the current scenario taking into account that the real-time RT-PCR test alone, cannot be relied upon for diagnosis of covid-19 as there is risk of obtaining false-negative and false-positive results. There are reports that suggest that many 'suspected' cases of covid-19 infection who had presented with clinical symptoms and radiological findings that were typical of covid-19 went undiagnosed because of a false negative RT-PCR test [17]. This goes to prove that, a negative result does not exclude the possibility of COVID-19 infection and the diagnosis of covid-19 should be made after a comprehensive evaluation that includes keeping the clinical and radiological aspects in mind and correlating them with both Rapid antigen and RT-PCR test reports.

The use of gloves and face masks has not proved to be enough for protection and hence we recommend the use of Powered Air Purifying Respirator Devices for all otolaryngology procedures during this time. During the first wave of the pandemic, it was observed that while both $\mathrm{N} 95$ masks and tight-fitting goggles and use of powered air-purifying respirators (PAPRs) was recommended for safety of ENT surgeons $[18,19]$ they both had their respective advantages and disadvantages. Though, the PAPRs have their own disadvantages of being cumbersome to use and limiting visibility due to fogging up amongst many others [20] we would strongly suggest using them for all otolaryngology procedures currently as they offer better protection. This is in accordance with adocument by National Services Scotland [21], that recommends the use of a Filtering face piece 3 (FFP3) respirator and eye protection for all aerosol generating procedures. Their use both for diagnostic and treatment procedures is advisable for the healthcare staff irrespective of whether patients are RT-PCR positive or not.

The same document by the National Health Services, Scotland, describes in detail the levels of Personal Protective Equipment (PPE) for Healthcare Workers and we recommend the use of Level 3 Enhanced PPE that includes fluid-resistant long-sleeved surgical gowns, full length disposable plastic aprons, FFP3 respirator or powered hood respirators and Disposable boot covers among others for all otolaryngology or upper airway procedures during these times [21]. 
Only emergency ear surgeries must be undertaken and the ones requiring drilling must be carried out using enhanced level 3 PPE. Studies suggest restricting the use of high speed drills during these times or reduce their speed [17] as high speed drills have a tendency to generate contaminated aerosols [22,23]. In accordance with certain studies, we would like to recommend the use of endoscope over microscope and operate under plastic drapes as these practices would help prevent face and eye contamination while carrying out a ear surgery [16].

For any upper airway and sinonasal procedures and while performing surgical debridement in cases of mucormycosis we recommend the use of a transparent plastic drapes while carrying out endoscopic procedures during this time. While we believe that powered instrumentation should be avoided during this time, in cases where it is required to be done and for any other endoscopic procedures, studies advocate that the procedure be undertaken through small holes or fenestrations made in plastic drapes to limit the spread of aerosols [24].

These suggestions can prove to be helpful in the management of cases of mucormycosis that are being reported following covid-19 recovery. Despite the fact that mucormycosis is mostly being seen in patients after they have recovered from covid-19, a risk of reinfection by the virus still persists and hence taking all precautions while diagnosing and treating these patients including ascertaining their covid-19 status is advocated.

Studies suggest that Alternative surgical approaches like external paracanthal, paralateronasal, and sublabial approaches should be preferred over endoscopic approach during these times as they will limit the use of drills and microdebrider thereby minimizing the risk of infection by contaminated aerosols. instead of endonasal approach [25].

In our opinion, procedures like diagnostic nasal endoscopies and laryngoscopies should be avoided during this time. They must only be carried out in only cases of suspected malignancy, foreign body removal or while diagnosing and surgical debridement of case of mucormycosis that are being seen lately. While strict precautions for all procedures of general anesthesia are advocated, all procedures with microdebrider must be avoided during this time.

Smoke evacuation system for evacuating any smoke produced during the surgery is advocated [26].

\section{Conclusion}

There is enough evidence to suggest that the second wave of covid-19 has impacted the healthcare system of India in a big way with otolaryngologists being in the frontline since the start of the pandemic. The risk of acquiring the infection and succumbing to it is far greater than in the first wave of the pandemic. Moreover, there is an additional burden on the Otolaryngologists due to a sudden surge in cases of mucormycosis, for which they are again in the frontline. Hence a revised triage strategy and stricter levels of precaution are the need of the hour. Dealing with every patient as a covid positive patient is suggested until the covid status is ascertained by a comprehensive evaluation that includes clinical, radiological and Rapid antigen and RT-PCR testing. Protecting the healthcare staff should be considered an absolute priority.

\section{Funding}

Not applicable.

\section{Compliance with Ethical Standards}

Approval from the ethical committee was not required for this study. I confirm that the manuscript has not been published or submitted for publication to any other journal.

\section{Conflicts of Interests}

The authors declare that they have no conflicts of interests.

\section{Informed Consent}

Not applicable.

\section{Bibliography}

1. Chang D., et al. "Protecting health-care workers from subclinical coronavirus infection". The Lancet Respiratory Medicine 8.3 (2020): e13.

2. Why Surgeons Don't Want to Operate Right Now - BNN Bloomberg. BNN (2020).

3. Pedro S., et al. "Conditions for a Second Wave of COVID-19 Due to Interactions Between Disease Dynamics and Social Processes". Frontiers in Physics (2020): 8.

4. Chetterje P. “Gaps in India's preparedness for COVID-19 control”. The Lancet Infectious Diseases 20.5 (2020): 544. 
5. Saraya A. "Indian response to COVID-19: Expertise and transparency”. Indian Journal of Public Health 64.6 (2020): 243.

6. MoHFW | Home. Mohfw.gov.in (2021).

7. Ing E., et al. "Physician deaths from corona virus (COVID-19) disease". Occupational Medicine (Chic Ill) 70.5 (2020): 370374 .

8. Davies N., et al. "Estimated transmissibility and impact of SARS-CoV-2 lineage B.1.1.7 in England". Science 372.6538 (2021).

9. Volz E., et al. "Assessing transmissibility of SARS-CoV-2 lineage B.1.1.7 in England". Nature 593.7858 (2021): 266-269.

10. Chen J., et al. "Mutations Strengthened SARS-CoV-2 Infectivity". Journal of Molecular Biology 432.19 (2020): 5212-5226.

11. Xu S and Li Y. "Beware of the second wave of COVID-19". The Lancet 395.10233 (2020): 1321-1322.

12. Huang C., et al. "Clinical features of patients infected with 2019 novel coronavirus in Wuhan, China". The Lancet 395.10233 (2020): 497-506.

13. COVID Live Update: $170,262,216$ Cases and 3,540,517 Deaths from the Coronavirus - Worldometer. Worldometers.information (2020).

14. Bai Y., et al. "Presumed Asymptomatic Carrier Transmission of COVID-19". JAMA 323.14 (2020): 1406.

15. Coronavirus Disease 2019 (COVID-19). Centers for Disease Control and Prevention (2021).

16. Bhardwaj R., et al. "Covid-19 and Ear Surgery: Treatment Strategies and Triage during the Post-lockdown Period". Indian Journal of Otolaryngology and Head and Neck Surgery (2021).

17. Wang Y., et al. "Combination of RT-qPCR testing and clinical features for diagnosis of COVID-19 facilitates management of SARS-CoV-2 outbreak". Journal of Medical Virology 92.6 (2020): 538-539.

18. Tay J., et al. "Surgical Considerations for Tracheostomy During the COVID-19 Pandemic". JAMA Otolaryngology-Head and Neck Surgery 146.6 (2020): 517.

19. Coronavirus Disease (COVID-19) Situation Reports. WHO International (2021).
20. Givi B., et al. "Safety Recommendations for Evaluation and Surgery of the Head and Neck During the COVID-19 Pandemic". JAMA Otolaryngology-Head and Neck Surgery 146.6 (2020): 579.

21. Nipcm.hps.scot.nhs.uk.https://www.nipcm.hps.scot.nhs.uk/ media/1437/2019-02-11-aide-memoire-for-levels-of-personal-protective-equipment-ppe-for-healthcare-workers-forpatient-care.pdf.

22. Heikkinen T., et al. "Prevalence of Various Respiratory Viruses in the Middle Ear during Acute Otitis Media." New England Journal of Medicine 340.4 (1999): 260-264.

23. Jewett D., et al. "Blood-Containing Aerosols Generated by Surgical Techniques: A Possible Infectious Hazard". American Industrial Hygiene Association Journal 53.4 (1992): 228-231.

24. Workman A., et al. "Endonasal instrumentation and aerosolization risk in the era of COVID-19: simulation, literature review, and proposed mitigation strategies". International Forum of Allergy and Rhinology 10.7 (2020): 798-805.

25. He F., et al. "Coronavirus disease 2019: What we know?" Journal of Medical Virology 92.7 (2020): 719-725.

26. Juvekar M and Sarkar B. "Guidelines for otorhinolaryngologists and head neck surgeons in coronavirus disease 2019 pandemic". The Egyptian Journal of Otolaryngology 37 (2021): 22.

\section{Volume 3 Issue 12 December 2021} (c) All rights are reserved by Akriti Sharma., et al. 